\title{
Top Yukawa New Strong Sector, Higgs Decay and Production
}

\author{
Holger Bech Nielsen* ${ }^{*}$ \\ Niels Bohr Institue, Blegdamsvej 15-21, DK2100 Copenhagen Ø \\ E-mail: hbech@nbi.dk, hbechnbi@gmail.com
}

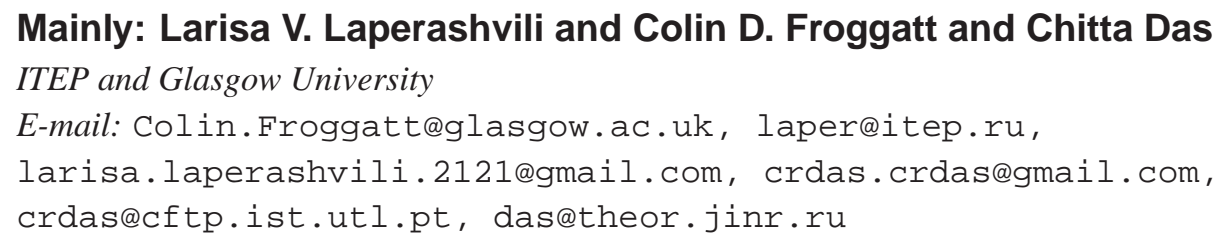

Mainly: Larisa V. Laperashvili and Colin D. Froggatt and Chitta Das

ITEP and Glasgow University

E-mail: Colin.Froggatt@glasgow.ac.uk, laper@itep.ru,

larisa.laperashvili.2121@gmail.com, crdas.crdas@gmail.com,

crdas@cftp.ist.utl.pt, das@theor.jinr.ru

\begin{abstract}
We call attention to the fact that the top-Yukawa-coupling $g_{t}$ being of order unity a priori opens up the possibility for there existing a new sector of strongly interacting particles. That is to say that you may have phenomena such as bound states among the particles involved in these relatively strong interacions caused by the Yukawa coupling $g_{t}$, much analogous to QCD. That is to say: top quarks and Higgses should form possible resonances and strong binding. Really one should rather ask for whether $g_{t}^{2} / 2 /(2 \pi)$ is large or small compared to unity, and thus it seems at first not of order unity, but if we correct by a factor 12 for considering a bound state of 12 constituents, we get a number of order unity, and a strong sector is expected! After the Korfu meeting a new diphoton resonace of mass $750 \mathrm{GeV}$ were found in ATLAS and CMS, which could potentially be a bound state of 6 top +6 anti top binding strongly, precisely because the top-Yukawa coupling is "large".
\end{abstract}

Proceedings of the Corfu Summer Institute 2015 "School and Workshops on Elementary Particle Physics and Gravity"

1-27 September 2015

Corfu, Greece

\footnotetext{
* Speaker.

${ }^{\dagger}$ Article is written by me Nielsen, but the originating ideas are from works with Colin Froggatt, Larisa Laperashvili Chitta Das etc. although I here seek to look at them in a more genralized way.
} 


\section{Introduction}

\subsection{Could the Strong Top Yukawa Coupling Open a New Sector of Strong Interactions inside the Standard Model ?}

We want to argue that there is the possibility, that the top quark Yukawa coupling to the Higgs $g_{t}=0.93_{5}$ is so large, that building up Feynman diagrams involving mainly this coupling would cause the perturbation series to converge so badly that the result would be uncontrollable in a similar way as phenomena (confinement say) that occur in Q.C.D. which are not understandable perturbativly.

If the Yukawa coupling $g_{t}$ is not "small" we cannot trust perturbation theory, and non-perturbative phenomena as phase transitions, bound states,etc. ... can occur.

Observing them experimentally we might at first take such non-perturbative effects for being "new physics"!
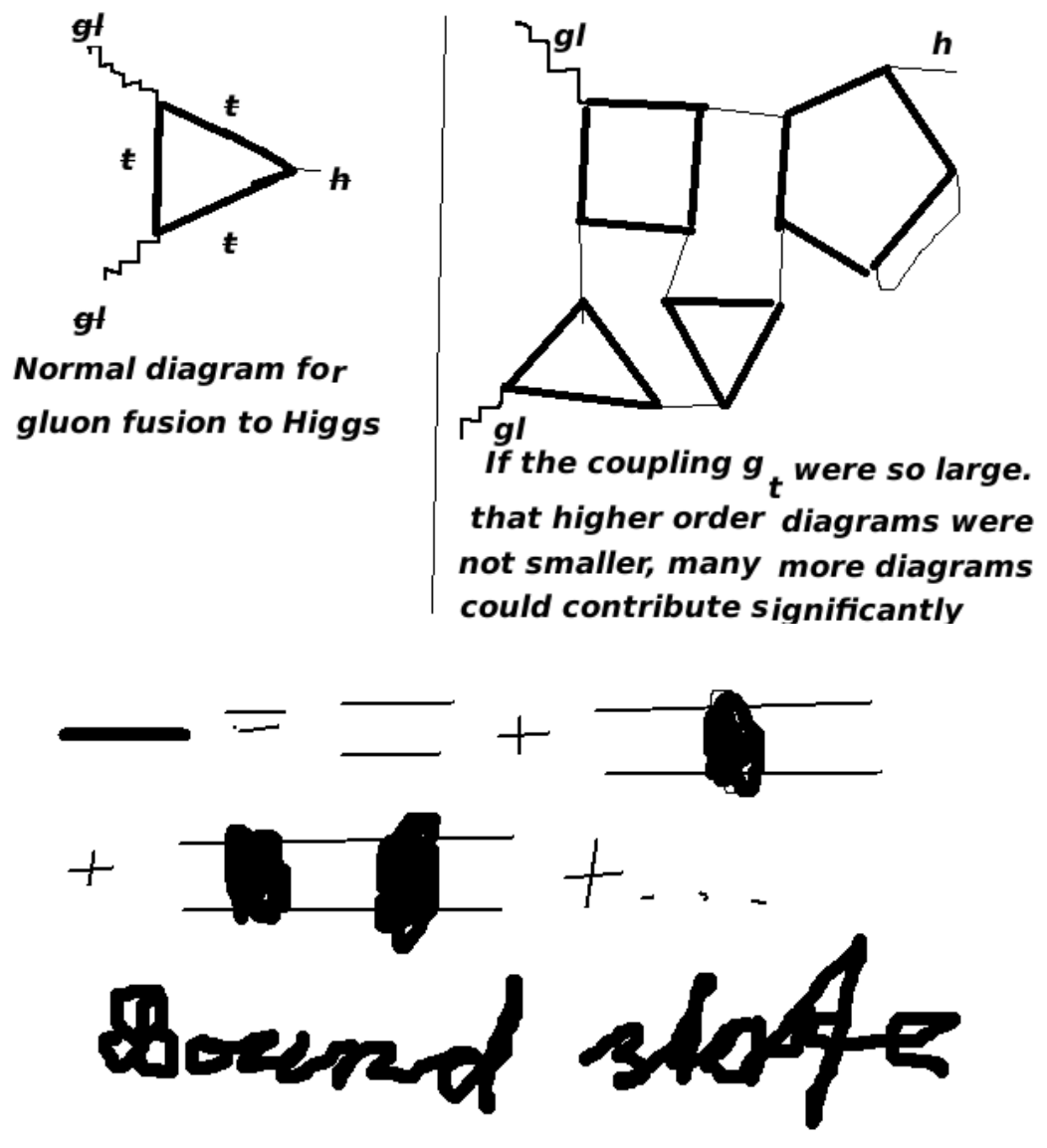

Once there may be binding between top-quarks say due to Higgs exchange, it will be more efficient to bind so many as allowed by Pauli principle - or filling a shell - and not just two: Rather we have for long time dreamt about a bound state[15, 16, 17, 18, 19, 20, 21, 22, 23, 24, 25, 26, 27, ?] of 6 top +6 antitop, because it is a closed shell system. 
There is an only two standard deviation little peak in the Higgs + Higgs mass spectrum with a mass $\sim 300 \mathrm{GeV}$, which could a priori be resonace due to the new type of a strong interaction, which we here claim. It were especially impressive to us in as far as Colin D. Froggatt and I in the appendix of an article [28] in a slightly indirect way came to a very crude estimate for the mass of our bound state of 6 top +6 anti top quarks of $285 \mathrm{GeV}$. According to the mentioned philosophy, that potential bound states should be in their binding dominated by the exceptionally strong top Yukawa coupling, the most promissing place to look for our type of resonace would at first seem to be in top or Higgs involving decays, and thus looking in Higgs + Higgs decay is extremely suggestive.

Remark added after the conference: The very little fluctuation in the Higgs + Higgs spectrum has hardly any chance of being anything but a fluctuation, but the diphoton bumb seen after the conference but before finishing the proceedings at mass $=750 \mathrm{GeV}$ looks more promissing. So at this moment of writting the believe is that it is the $750 \mathrm{GeV}$ diphoton peak which should be a bound state due to the strong interaction due to the stength of the top-Yukawa-coupling[29].

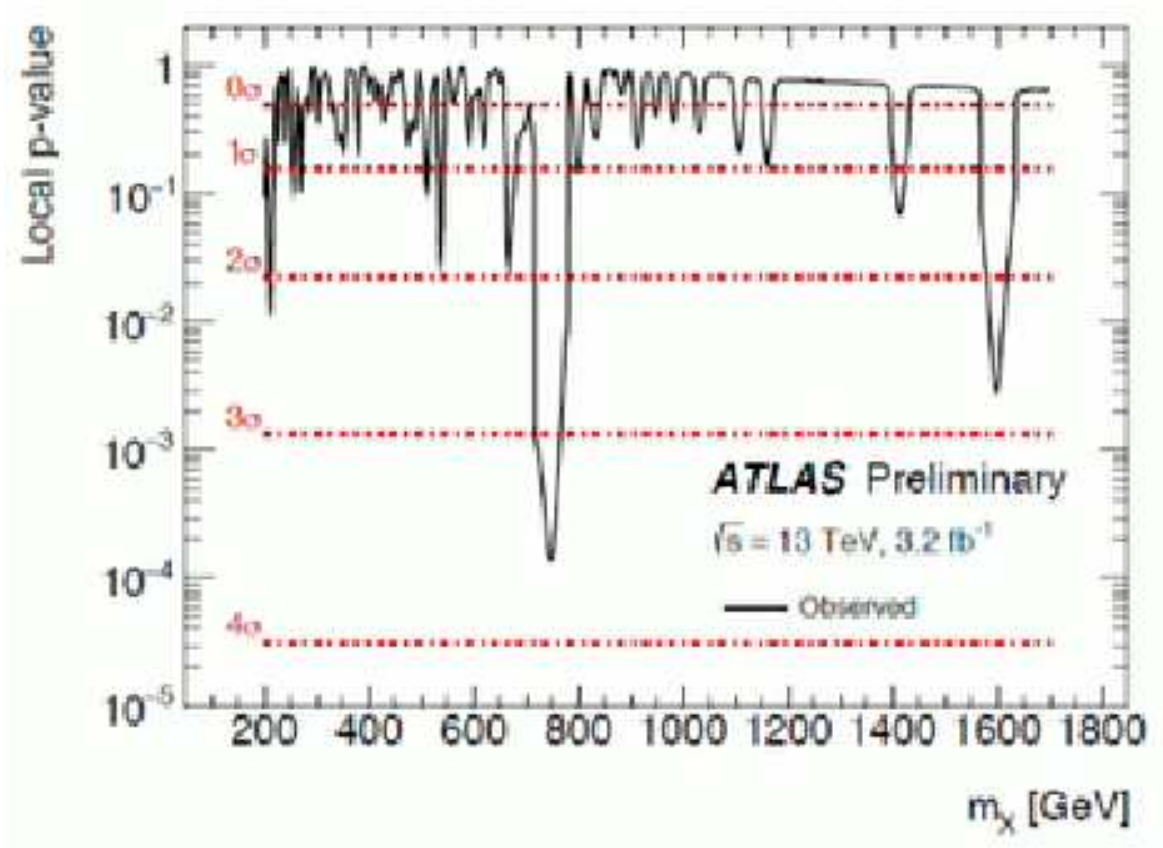

The retorical question in the situation, wherein the Standard Model functions so extremely well: If Standard Model works so well there might be little place for genuine "new physics" but could there be some effective new physics inside the Standard Model as e.g. due to non-perturbative regions in phase space (a second q.c.d. so to speak)? What one could realistically hope for:

- Bound states or resonaces formed from a may be relatively large number of the particles involved in the hoped for large coupling $g_{t}$. I.E. bound states or resonances formed from top or Higgs.

- Or some phase transition, so that there e.g. would be another vacuum phase. Actually we have some model/speculations: How to make dark matter from such a new phase.[1, 2, 3, 4, $5,6,7,8,9]$ 


\section{Is say $g_{t}$ Large or Small or On Border?}

- At first it happens to be $g_{t}=.93_{5}$ so indeed $g_{t} \approx 1$,

- But when evaluating Feynman diagrams there appear for every loop "following the momentum integrations" a denominator $(2 \pi)^{4}$, and making e.g. Gaussian approximation one gets extra $\pi^{2} * 2^{2 / 3} / 3$, and actually the factor $1 /\left(16 \pi^{2}\right)$ is wellknown to appear, in fact for each loop.

- in this light - since $16 \pi^{2} \approx 160$ - we cannot claim but that $g_{t}^{2} /\left(16 \pi^{2}\right)$ is much smaller than unity. So we get for each loop a small factor and perturbation should be o.k.

- But now we must take into account that because the top-quark has spin and color there must appear a factor of the order $N_{c} * 2$ (or4) for each top quark loop so that we rather have for an average loop - which say could be made one out of 3 loops - a factor $\left(N_{c} * 2(\text { or } 4)\right)^{1 / 3} \approx 2$

- Also the number of Feynman diagrams increase at the end rather drasticly, like factorial.

\section{Gluon-Photon-Relation}

\section{Rules, that may be Tested}

Note we have only the one coupling $g_{t}$, except if we decorate by gluons and thus the type of diagrams that can come into the strong coupling - in the new strong sector - is only this single Yukawa coupling! This must be used again and again in the Feynman digrams working for the strong coupling effects. This gives the prediction that that

- 1. The resonances or bound states belonging to this new strong sector would dominantly couple to Higgs and to top. E.g. a decay of a resonance in the scheme could be to a couple of Higgs bosons. (or into a couple of top anti top say).

- 2. Coupling externally to gluons (pairs) or to photons could only be arranged by the coupling attaching to the top-quark and thus would happen in just the ratio of the top-couplings to respectively gluon and photon. (It is this relation that is a prediction characteristic of our scheme; it means it is only the top-coupling correction $\kappa_{t}$ which is changed effectively when we look at Higgs decay and production.) 


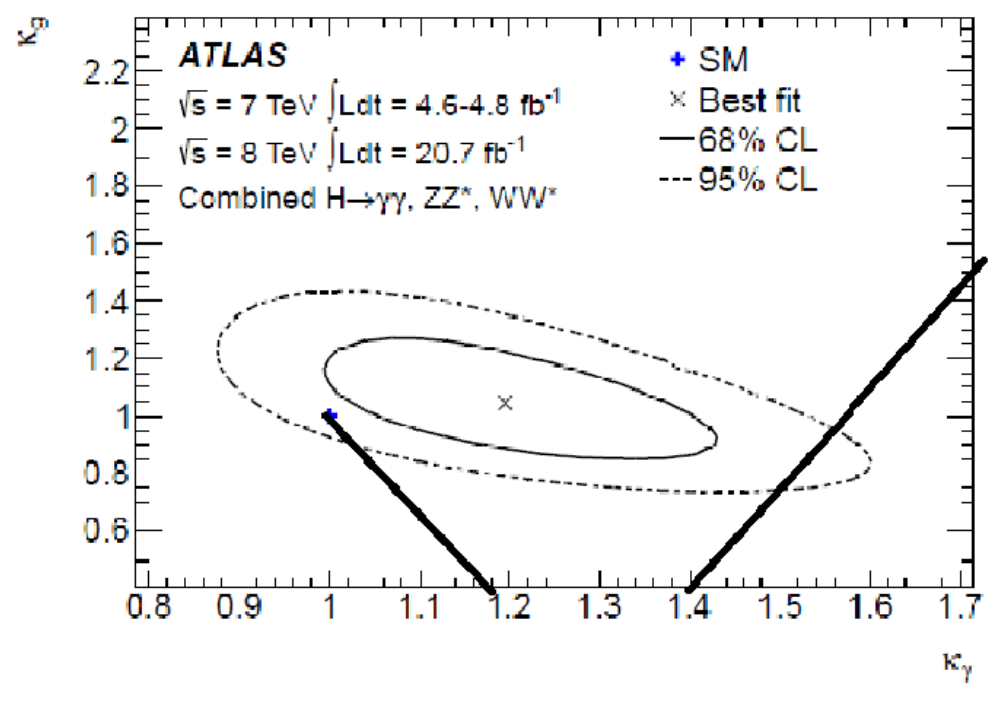

The present considerations of a strong interaction sector or almost equivalently some bound state modifying the Higgs decay to two photons or the strongly related coupling of the Higgs to a pair of gluons, with the gluons being analogous to the photons is indeed found in our earlier work [30].

If we assume, that there is some correction to the Higgs effective couplings $H \gamma \gamma$ and $H g g$ (where $g$ denotes gluon) both comming from a lot of very high order Feynman diagrams only involving Higgs $H$ and top $t$ propagators, then the effective contributions to these two couplings causing deviations from the ("naive") Standard model - and thus to $\kappa_{\gamma}-1$ and $\kappa_{g}-1$ - must be in a ratio given by the ratio of the coupling of a photon relative to that of gluons to the top quark. On next figure I inserted a line in the $\kappa_{g}$ versus $\kappa_{\gamma}$ plane (the plane of the foregoing figure) corresponding to the prediction for such a strong coupling effect.

Although the actually first seen fluctuation deviating from Standard Model of an excess decay rate to $\gamma \gamma$ were largely wash out by CMS. we still can get a tiny bit better fit - with one more parameter though - by our curve/line presenting some strong coupling effect involving only top and Higgs than by pure Standard Model $\left(\kappa_{F}=1, \kappa_{V}=1\right)$. Our best fit means $\kappa_{F}=0.9$ or the $g g H$ - which is mainly given by the top loop - being multiplied by 0.9 compared to the Standard Model. Hereby the gluon fusion amplitude goes down by $10 \%$ and so the rate by $20 \%$. There are no top-quark decay of the Higgs for kinematical reasons. So the decays which are not only due to loops will not be significantly influenced. But the $\gamma \gamma$ decay is given by a negative interference between boson loops and the top-loop mainly in the Standard Model. Therefore an effect formally diminishing the top-coupling - and thereby the top-loop - from 1 to .9 say, will increase the decay rate of the Higgs into the $\gamma \gamma$ channel. With the restriction that only diagrams with top and Higgs alone as propagators only using the vertex top-yukawa a decrease of the $\kappa_{t} \approx \kappa_{F}$ to 0.9 (from the Standard Model 1) would let $\kappa_{\gamma} 1.3_{5}$ meaning then that the decay rate into $\gamma \gamma$ for the Higgs would go up by $1.03_{5}^{2}=1.07$ i.e. by $7 \%$. 

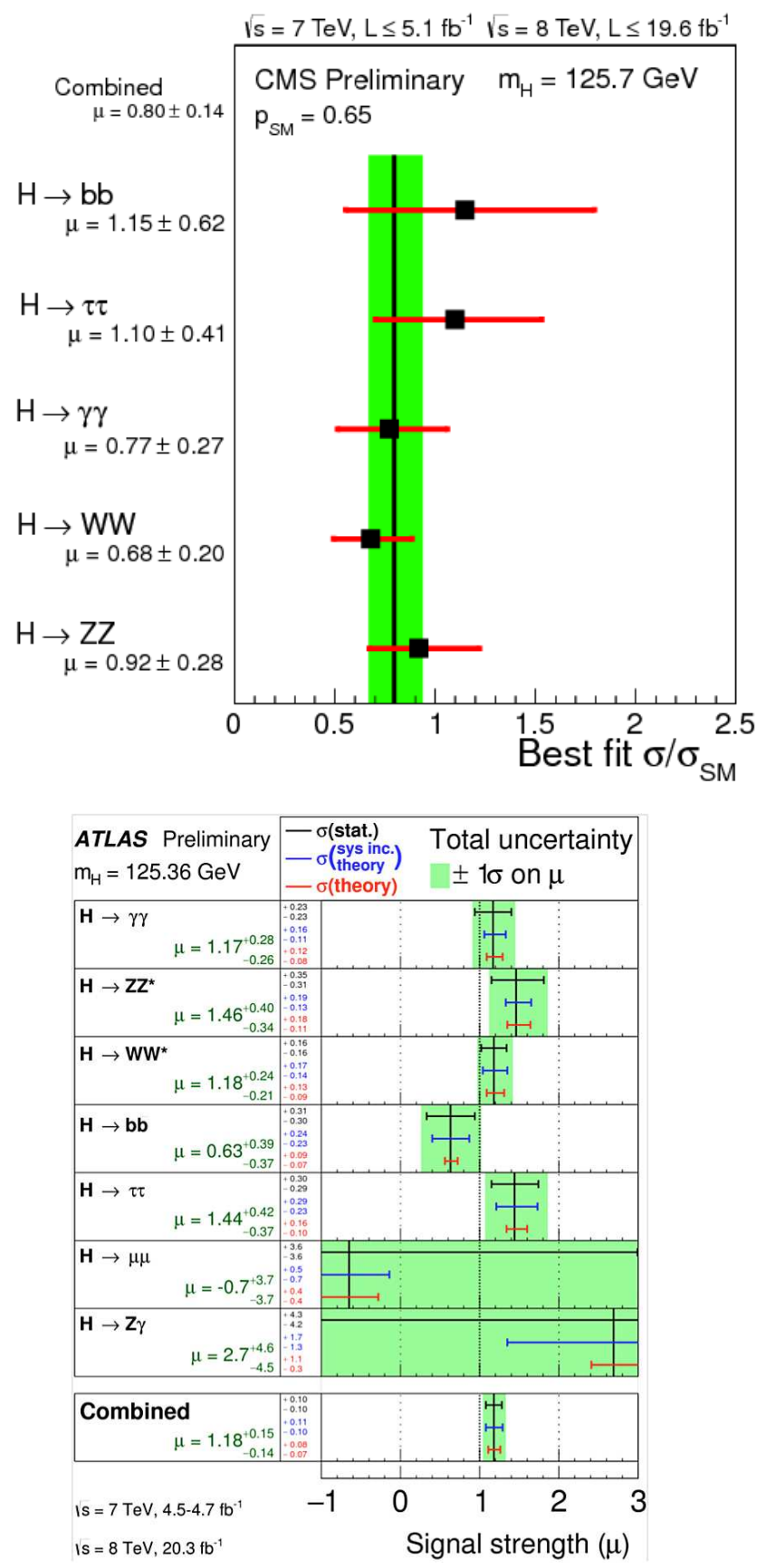


\section{Calculation with Bound State}
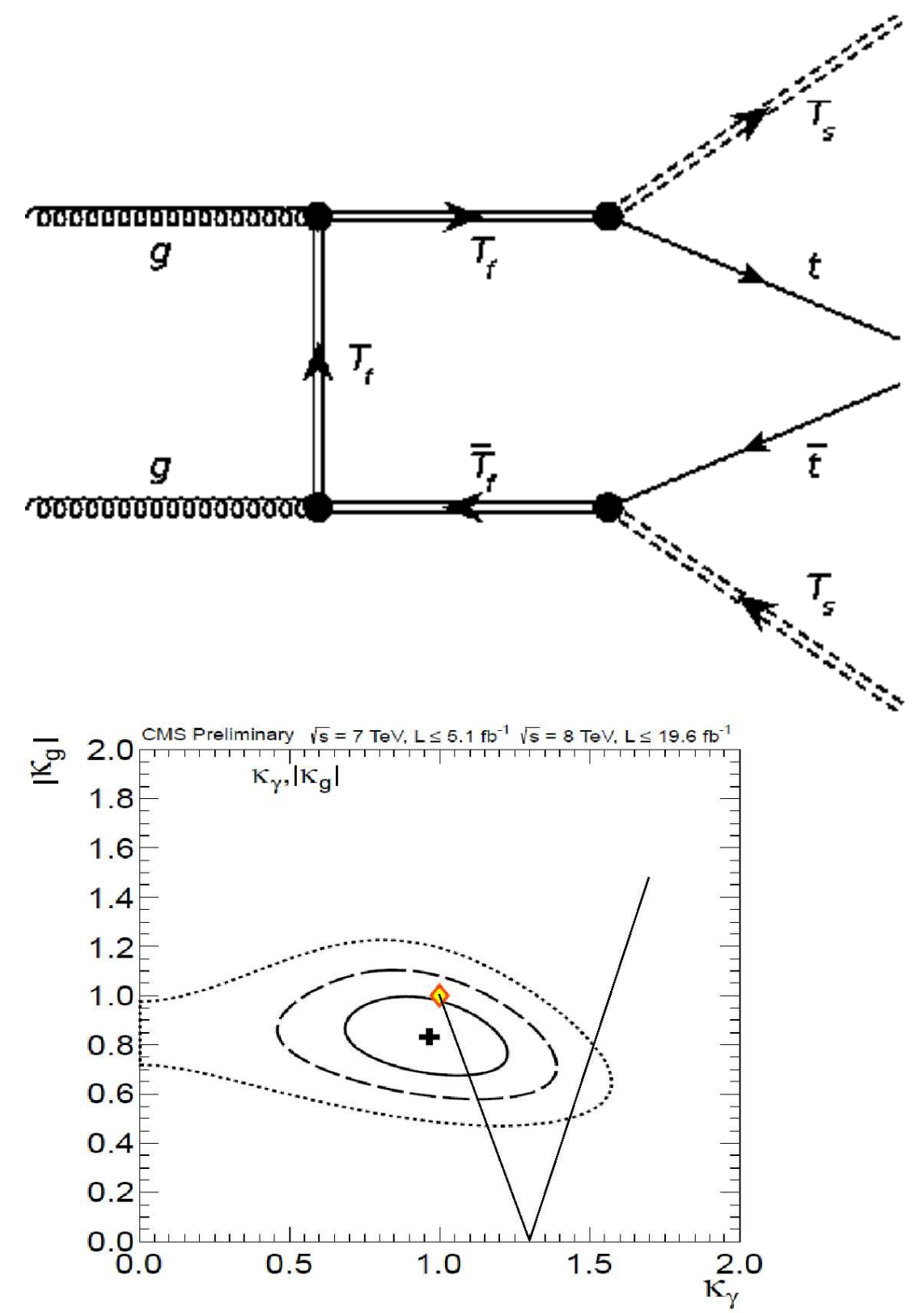

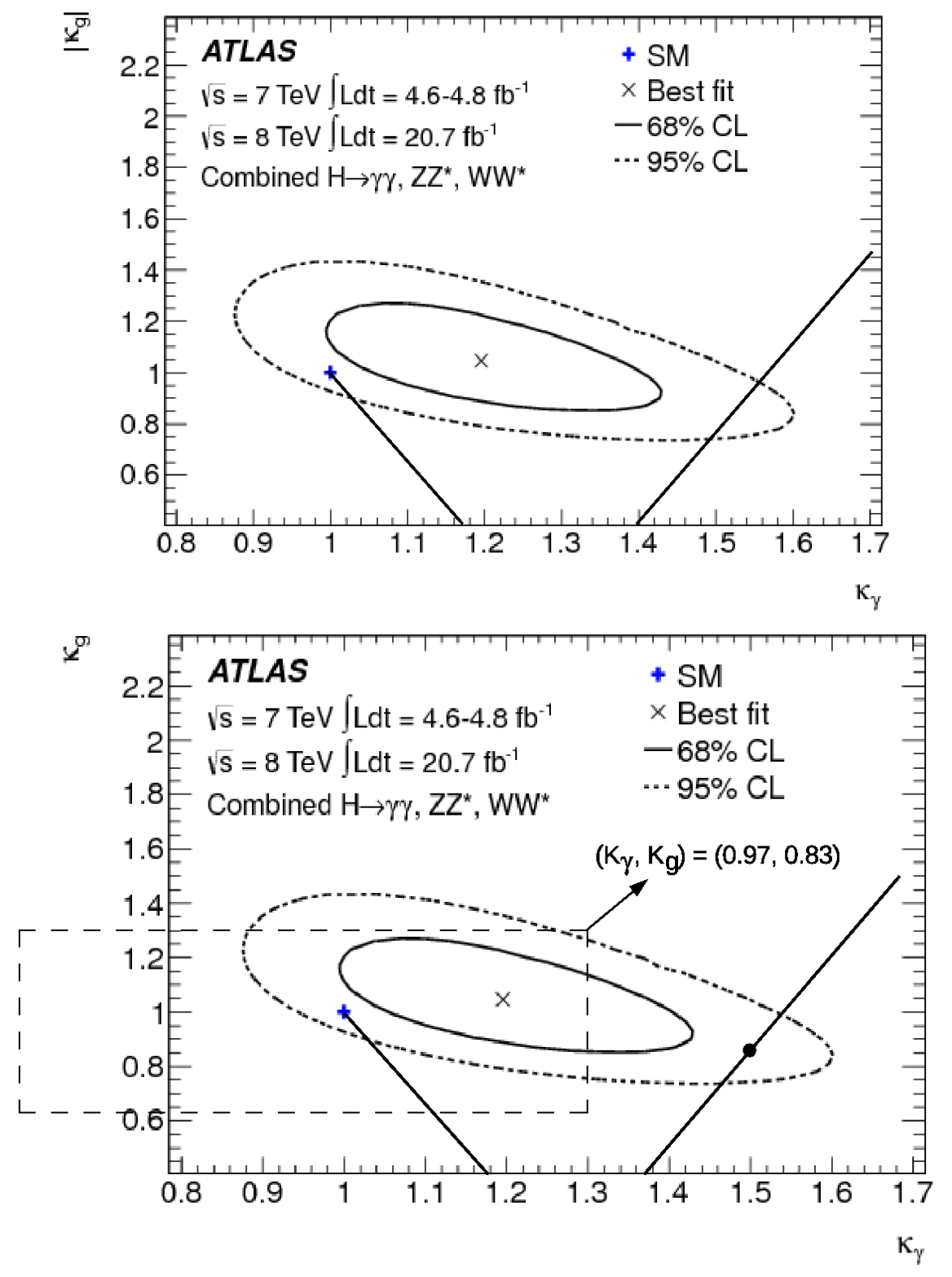

Some typical values for the hard estimate of the mass of the bound state $S$, which at first must be "small compared to the mass of the constituents" 12 top quarks having collective mass $12 \cdot 173 \mathrm{GeV}=2.1 \mathrm{TeV}$, and sufficiently large that the Higgs boson cannot decay just into the two $S$-particles, are put forward in the Table 1 here. A mass $m_{S}>m_{H} / 2=63 \mathrm{GeV}$ is required because a smaller $S$-mass would have made the Higgs decay dominantly into pairs of our bound state, and no Higgses would be found in the ordinary channels so far. 


\begin{tabular}{|c|c|c|c|c|}
$m_{S}$ & $K$ & $\kappa_{\gamma}$ & $\kappa_{g}$ & Agreement \\
\hline \hline $140 \mathrm{GeV}$ & 0.864 & 1.52 & 0.8 & $\kappa_{\gamma}$ agrees ATLAS, $\kappa_{g}$ more CMS \\
\hline $280 \mathrm{GeV}$ & 0.216 & 1.13 & 0.55 & $\kappa_{\gamma}$ agr. ATLAS \\
\hline $560 \mathrm{GeV}$ & 0.054 & 1.03 & 0.9 & CMS and ATLAS \\
\hline
\end{tabular}

\section{Outlook}

\section{Outlook on Hopes to use New Strong Sector in Standard Model (alone!)}

The speculative hopes you could have to solve some of the problems of the Standard Model could be:

- 1. A new phase - as could easily happen if the coupling is close to the diagram series diverging barely(changing it a bit lets the effects of high order come in or not)-. Then one phase could be the usual vacuum, while the other phase could be "vacuum" realized inside macroscopic bodies, which filled with ordinary matter under pressure could be macrosopic balls making up the dark matter!

- 2. Since the top-Yukawa coupling like e.g. the $\alpha_{S}(\mu)$ runs due to the renormalization group, it will pass the "critical value" for being so strong that it makes severe non-perturbative effects at a certain scale $\mu_{\text {crit }}$. This could at least formally point to a way, in which one could formally obtain a number for even the scale problem number: the ratio of the planck scale to the weak scale $v / M_{p l}$.

In fact the fact that the running yukawa coupling $g_{t}(\mu)$ takes on a value very close to what we have argued is just between it being large and small (it is in fact on the border-line of making the diagram series diverge practically) just at the weak scale, i.e. $g_{t}\left(M_{Z}\right)$ is just on the border to make the diagram contributions increase with the order.) means that the weak scale is pointed out as the scale at which the top-yukawa is just passing the critical value $(\approx 1)$.

\section{Conclusion}

\section{Conclusion and Resume}

- The top Yukawa coupling $g_{t}$ could be so strong that it means there is a new strong interaction section with strongly bound states new phases etc.

- Such a new strong sector could if ignored look like and have effects like the new physics we dream about finding in LHC soon.

- A characteristic signal is that it should seemingly show up as a top-coupling correction $\kappa_{t}$ (or crudely $\kappa_{F}$ in the plot shown to you), because it is only diagrams with top and Higgs that are in this system of new strong sector. 
- Actually this just change the $\kappa_{t}$ can fit very well with the Higgs boson decay data; but of course the Standard Model is still also fitting in what we call the "naive" (= perturbative) approximation.

\section{Conclusion and Resume continued}

- If we believe we could crudely estimate our concretization by a bound state of 6 top and 6 anti top quarks we could even predict the strength of the strong sector effect. The best fitting mass for this bound state is only a factor 2 than a crudely estimated value publishedin an appendix. But with calculation it ought to be possible to calculate the size of the effect and thus obtain a correction to the Higgs decay branching ratios and production rate without any new parameters.

- Such a new strong sector could quite similarly to "new physics" providing new particles contribute some bound states or resonaces that could at least approximately be treated as new particles.

- The new strong coupling sector could also easily be speculated to provide a new phase.

- Such a new phase might give rise to macrosopic objects with this phase inside them. Such objects could perhaps make up the dark matter. (Usually one says that dark matter is completely excluded in the pure Standard Model, so even if a bit complicated a pure Standard Model picture of dark matter would be an achievement!)

\section{Acknowledgement}

HBN wishes to than the Niels Bohr Institute for support and for allowance to stay as emeritus. Og course I also thank the collaborators with whom the presented work were done and for their discussions.

\section{References}

[1] D.L. Bennett and H.B. Nielsen, Int. J. Mod. Phys. A9, 5155 (1994); arXiv:hep-ph/9311321; ibid., 14, 3313 (1999); arXiv:hep-ph/9607278.

[2] D.L. Bennett, C.D. Froggatt and H.B. Nielsen, in Proceedings of the 27th Int. Conf. on High Energy Physics, Glasgow, Scotland, 1994, eds. P. Bussey and I. Knowles (IOP Publishing Ltd., 1995), p. 557; in Perspectives in Particle Physics 94, eds. D. Klabučar, I. Picek and D. Tadić (World Scientific, Singapore, 1995), p. 255; arXiv:hep-ph/9504294.

[3] C.D. Froggatt and H.B. Nielsen, Phys. Lett. B368, 96 (1996), arXiv:hep-ph/9511371.

[4] L.V. Laperashvili, Yad. Fiz. 57, 501 (1994) [Phys. Atom. Nucl. 57, 471 (1994)].

[5] C.D. Froggatt, L.V. Laperashvili, R.B. Nevzorov and H.B. Nielsen, Phys. Atom. Nucl. 67, 582 (2004) [Yad. Fiz. 67, 601 (2004)]; arXiv:hep-ph/0310127. 
[6] C.D. Froggatt, L.V. Laperashvili and H.B. Nielsen, Phys. Atom. Nucl. 69, 67 (2006), arXiv:hep-ph/0407102.

[7] D.L. Bennett, L.V. Laperashvili and H.B. Nielsen, Proceedings of the 9th Workshop on What Comes Beyond the Standard Models, Bled, Slovenia, 16-26 September, 2006, eds. M. Breskvar et al. (Bled workshops in physics, Vol.7, No. 2, DMFA, Zaloznistvo, Ljubljana, 2006), p. 10, arXiv:hep-ph/0612250; Proceedings of the 10th Workshop on What Comes Beyond the Standard Model, Bled, Slovenia, 17-27 September, 2007, eds. M. Breskvar et al. (Bled workshops in physics, Vol.8, No. 2, DMFA-Zaloznistvo, Ljubljana, Dec. 2007), arXiv:0711.4681.

[8] C.D. Froggatt, R.B. Nevzorov and H.B. Nielsen, Smallness of the cosmological constant and the multiple point principle, 2007 Eur. Conf. on High Energy Physics, Manchester, England, 19-25 July, 2007, J. Phys. Conf. Ser. 110, 072012 (2008), arXiv:0708.2907.

[9] C.D. Froggatt, R.B. Nevzorov, H.B. Nielsen and A.W. Thomas, Phys. Lett. B737, 167 (2014), arXiv:1403.1001; 2015 Eur. Phys. Society Conf. (EPS-HEP 2015), Vienna, Austria, 22-29 July, 2015, arXiv: 1510.05379 .

[10] C.D. Froggatt and H.B. Nielsen, Origin of Symmetries, World Scientific, Singapore, 1991.

[11] C.R. Das and L.V. Laperashvili, Int. J. Mod. Phys. A20, 5911 (2005), arXiv:hep-ph/0503138.

[12] B.G. Sidharth, The Thermodynamic Universe, World Scientific, Singapore, 2008; Int. J. Mod. Phys. A13, 2599 (1998), arXiv:quant-ph/9808031; Int. J. Th. Phys. 37, 1307 (1998); Found. Phys. 38, 695 (2008), arXiv:0704.1517.

[13] L.V. Laperashvili, H.B. Nielsen and B.G. Sidharth, arXiv:1503.03911.

[14] A. Das and B.G. Sidharth, EJTP 12 No. IYL 15-34, 139 (2015).

[15] C.D. Froggatt and H.B. Nielsen, Trying to understand the Standard Model parameters, invited talk by H.B. Nielsen at the "XXXI ITEP Winter School of Physics", Moscow, Russia, 18-26 Feb, 2003; Surveys High Energy Phys. 18, 55 (2003), arXiv:hep-ph/0308144.

[16] C.D. Froggatt, H.B. Nielsen and L.V. Laperashvili, Hierarchy-problem and a bound state of 6 t and 6 anti-t, in: Proceedings of Coral Gables Conference on Launching of Belle Epoque in High-Energy Physics and Cosmology (CG 2003), Ft. Lauderdale, Florida, 17-21 Dec, 2003.

[17] C.D. Froggatt, H.B. Nielsen and L.V. Laperashvili, Int. J. Mod. Phys. A20, 1268 (2005), arXiv:hep-ph/0406110.

[18] C.D. Froggatt and H.B. Nielsen, Phys. Rev. D80, 034033 (2009), arXiv:0811.2089.

[19] C.D. Froggatt and H.B. Nielsen, Hierarchy Problem and a New Bound State, in: Proceedings of the Eur. Conf. on What comes beyond the Standard Model? Symmetries beyond the Standard Model, p.73, Portoroz, Slovenia, 12-17 July 2003 (DMFA, Zaloznistvo, 2003), arXiv:hep-ph/0312218.

[20] C.D. Froggatt, The Hierarchy problem and an exotic bound state, in: Proceedings of the 10th Int. Symposium on Particles, Strings and Cosmology, (PASCOS 04), Boston, Massachusetts, 16-22 Aug, 2004. Published in: "Boston 2004, Particles, strings and cosmology", pp.325-334, arXiv:hep-ph/0412337.

[21] C.D. Froggatt, L.V. Laperashvili and H.B. Nielsen, A New bound state $6 t+6$ anti-t and the fundamental-weak scale hierarchy in the Standard Model, in: Proceedings of the 13th Int. Seminar on High-Energy Physics: QUARKS-2004, Pushkinskie Gory, Russia, 24-30 May, 2004, arXiv:hep-ph/0410243. 
[22] C.D. Froggatt, L.V. Laperashvili and H.B. Nielsen, Phys. Atom. Nucl. 69, 67 (2006) [Yad. Fiz. 69, 3 (2006)]; arXiv:hep-ph/0407102.

[23] C.D. Froggatt, L.V. Laperashvili, R.B. Nevzorov and H.B. Nielsen, The Production of $6 t+6 \bar{t}$ bound state at colliders. A talk given by H.B. Nielsen at CERN, 2008, preprint CERN-PH-TH/2008-051.

[24] C.D. Froggatt, L.V. Laperashvili, R.B. Nevzorov, H.B. Nielsen and C.R. Das, arXiv:0804.4506.

[25] C.D. Froggatt and H.B. Nielsen, New Bound States of several Top-quarks bound by Higgs Exchange, in: Proceedings of the 34th Int. Conf. on High Energy Physics (ICHEP 2008), 30 Jul - 5 Aug, 2008, Philadelphia, Pennsylvania, arXiv:0810.0475.

[26] C.R. Das, C.D. Froggatt, L.V. Laperashvili and H.B. Nielsen, Int. J. Mod. Phys. A26, 2503 (2011), arXiv:0812.0828.

[27] C.D. Froggatt, C.R. Das, L.V. Laperashvili and H.B. Nielsen, Yad. Fiz.

[28] C. D. Froggatt and H. B. Nielsen, “Tunguska Dark Matter Ball,” Int. J. Mod. Phys. A 30 (2015) no.13, 1550066 doi:10.1142/S0217751X15500669 [arXiv:1403.7177 [hep-ph]].

[29] L. V. Laperashvili, H. B. Nielsen and C. R. Das, "New results at LHC confirming the vacuum stability and Multiple Point Principle,” Int. J. Mod. Phys. A 31 (2016) no.08, 1650029 doi:10.1142/S0217751X16500299 [arXiv:1601.03231 [hep-ph]].

[30] C. D. Froggatt, C. R. Das, L. V. Laperashvili and H. B. Nielsen, "Diphoton decay of the Higgs boson and new bound states of top and antitop quarks,” Int. J. Mod. Phys. A 30 (2015) no.21, 1550132 doi:10.1142/S0217751X15501328 [arXiv:1501.00139 [hep-ph]]. 